\title{
Kısmi Metrik Uzaylarda İntegral Tip Büzülme İçin Sabit Nokta Teoremleri
}

Fixed Point Theorems for Integral Type Contraction in Partial Metric Spaces

\section{Vildan ÖZTÜRK*}

Artvin Çoruh Üniversitesi, Eğitim Fakültesi, Matematik ve Fen Bilimleri Eğitimi Bölümü, 08000, Artvin

• Geliş tarihi / Received: 16.02.2018 • Düzeltilerek geliş tarihi / Received in revised form: 02.05.2018 • Kabul tarihi / Accepted: 15.05 .2018

\section{Öz}

Bu çalışmada kısmi metrik uzaylarda, uzaklığı değiştiren fonksiyonlar aracılığıyla (E.A)-özelliğini sağlayan dönüşümler için integral tipli büzülme tanımlanmış ve bu tipteki büzülmeyi sağlayan dört dönüşüm için ortak sabit nokta teoremi ispatlanmıştır.

Anahtar kelimeler: İntegral Tipli Büzülme, Kısmi Metrik, Sabit Nokta

\section{Abstract}

In this work, integral type contraction by altering distance functions for mappings satisfying (E.A)-property was introduced and common fixed point theorems for four mappings satisfying this type contraction was proved.

Keywords: Integral Type Contraction, Partial Metric, Fixed Point

\footnotetext{
*Vildan Öztürk; vildanozturk84@gmail.com; Tel: (0543) 48420 61; orcid.org/0000-0001-5825-2030
} 


\section{Giriş}

Tam metrik uzaylarda, sabit nokta teori üzerine ilk çalışmalar 1922 yılında Banach ile başlamıştır. Banach (1922), aşağıda verilen ve Banach büzülme prensibi olarak bilinen teoremi ispatlamıştır. $\mathrm{Bu}$ teorem, sabit noktanın varlığını kanıtladığı gibi tekliğini ve nasıl bulunacağını da ifade eder.

\subsection{Teorem}

$(X, d)$ bir tam metrik uzay, $f: X \rightarrow X$ dönüşümü her $x, y \in X$ ve bir $\alpha \in[0,1[$ sayıs için $d(f x, f y) \leq \alpha d(x, y)$ eşitsizliğini sağlarsa $f$ dönüşümünün tek bir sabit noktası vardır ve her $x \in X$ için $y_{n}=f^{n} x$ dizisi sabit noktaya yakınsar (Banach 1922).

Sabit nokta teori, matematikte olduğu kadar fizik, biyoloji, ekonomi, mühendislik, ve bilgisayar bilimleri gibi pek çok alanda uygulanmıştır. Zamanla, Banach büzülme prensibinin problemleri çözmek için yeterli olmadığ 1 görülmüştür.

Khan, Swaleh ve Sessa (1984), uzaklığ değiştiren fonksiyonları tanımlamıştır. $\mathrm{Bu}$ yardımc1 fonksiyonlar aracılığıyla, Banach büzülme prensibinin bir genelleştirmesi olarak tam metrik uzaylarda zayıf büzülmeyi üretmiş ve zayıf büzülmeyi sağlayan bir dönüşümün sabit noktasının varlığını ve tekliğini ispatlamıştır. Ayrıca, araştırmacılar tarafindan uzaklığı değiştiren fonksiyonlar kullanılarak gerek tek bir dönüşümün sabit noktasını kanıtlayan gerekse birden fazla dönüşümün ortak sabit noktasının varlığını ve tekliğini kanıtlayan daha genel sonuçlar verilmiştir (Babu vd., 2007; Dutta vd., 2008; Naidu, 2003; Rhoades, 2001).

\subsection{Tanım}

$\psi:[0, \infty[\rightarrow[0, \infty[$ dönüşümü

(a) $\psi(0)=0$,

(b) sürekli ve monoton azalmayan

özelliklerini sağlarsa uzaklığı değiştiren fonksiyon olarak tanımlanır (Khan vd., 1984)

\subsection{Teorem}

$(X, d)$ bir tam metrik uzay, $\psi$ uzaklığı değiştiren fonksiyon ve $f: X \rightarrow X$ dönüşüm olsun. $f$ dönüşümü her $x, y \in X$ ve bir $c \in(0,1)$ sayısı için $\psi(d(f x, f y)) \leq c \psi(d(x, y)) \quad$ eşitsizliğini sağlarsa $f$ dönüşümünün tek bir sabit noktası vardır (Khan vd., 1984). Banach büzülme prensibinin diğer bir genelleştirmesi ise tam metrik uzaylarda Brianciari (2001) tarafindan verilen integral tipli büzülmedir.

\subsection{Teorem}

$(X, d)$ bir tam metrik uzay ve $f: X \rightarrow X$ dönüşüm olsun. $\omega:[0, \infty[\rightarrow[0, \infty[\quad$ Lebesque integrallenebilir, toplanabilir ve her $\eta>0$ için

$$
\int_{0}^{\eta} \omega(t) d t>0
$$

özelliğini sağlayan fonksiyon olmak üzere, her $x, y \in X$ ve bir $c \in$ ]0,1[ sayısı için

$$
\int_{0}^{d(f x, f y)} \omega(t) d t \leq c \int_{0}^{d(x, y)} \omega(t) d t
$$

eşitsizliği sağlanırsa $f$ dönüşümünün bir tek sabit noktası vardır (Brianciari, 2001).

Son yıllarda, metrik uzayların sabit nokta teori için yeterli olmadığı ve metrik uzaylardan daha geniş uzayların olabileceği görülmüsstür. Kısmi metrik uzaylar, metrik uzayların bir genelleştirmesi olarak Matthew tarafından tanımlanmış ve Banach büzülme prensibi bu uzaylara aktarılmıştır (Matthew 1992,1994). Kısmi metrik uzayların en önemli özelliği bir noktanın kendisine olan uzaklığının sıfırdan farklı olabileceğidir. Bu özellikten dolayı yakınsaklık, süreklilik, tamlık gibi kavramlar metrik uzaylardakinden farklil1k göstermekte ve en önemlisi de bir dizinin yakınsadığı nokta tek olmak zorunda değildir. Sabit noktanın varlığını ve tekliğini ispatlamak için yakınsaklığın tek olması önemlidir. Matthew $(1992,1994)$ bu sorunu çözmek için kısmi metrik ile metrik arasında bir bağıntı vermiştir. Ayrıca, pek çok araştırmacı tarafından kısmi metrik uzaylarda çeşitli sabit nokta teoremleri ispatlanmıştır. (Abdeljawad, 2011; Altun vd., 2010; Kadelburg vd., 2013; Karapınar, 2011; Turkoglu vd., 2012; Valero, 2005).

\section{Temel Bilgiler}

\subsection{Tanım}

$X$ boş olmayan bir küme ve $p: X \times X \rightarrow[0, \infty[$, bir dönüşüm olsun. Her $x, y, z \in X$ için (p1) $x=y \Leftrightarrow p(x, x)=p(x, y)=p(y, y)$, 
(p2) $p(x, x) \leq p(x, y)$,

(p3) $p(x, y)=p(y, x)$,

(p4) $p(x, y) \leq p(x, z)+p(z, y)-p(z, z)$,

şartları sağlanırsa $(X, p)$ ikilisine kısmi metrik uzay denir (Matthew, 1994).

(p1) ve (p2) özelliğinden, $p(x, y)=0$ ise $x=y$ dir. Fakat, $x=y$ iken $p(x, y)=0$ olmak zorunda değildir (Matthew, 1994). Dolayısıyla, her kısmi metrik bir metrik değildir.

\section{2. Örnek}

$p:[0, \infty[\times[0, \infty[\rightarrow[0, \infty[, p(x, y)=\max \{x, y\}$ olarak tanımlanan dönüşüm bir kısmi metriktir. Fakat metrik değildir (Matthew 1994). Gerçekten; $x, y \in[0, \infty[$ için $x \neq 0, y \neq 0$ ve $x=y$ olsun. Bu durumda $p(x, y)=\max \{x, y\} \neq 0$ elde edilir.

\subsection{Tanım}

$(X, p)$ kısmi metrik uzay, $x \in X$ ve $\varepsilon>0$ bir reel sayı olsun. Bu durumda

$B_{p}(x, \varepsilon)=\{y \in X: p(x, y)<p(x, x)+\varepsilon\}$

kümesine $x$ merkezli $\varepsilon$ yarıçaplı açık yuvar,

$\overline{B_{p}}(x, \varepsilon)=\{y \in X: p(x, y) \leq p(x, x)+\varepsilon\}$

kümesine de $x$ merkezli $\varepsilon$ yarıçaplı kapalı yuvar denir (Matthew, 1994).

Matthew (1994), metrik ile kısmi metrik arasındaki bağıntıyı aşağıdaki önermeyle vermiştir.

\section{4. Önerme}

$(X, p)$ kısmi metrik uzay olsun. $p^{s}, p^{w}: X \times X \rightarrow$ $[0, \infty[$,

$p^{s}(x, y)=2 p(x, y)-p(x, x)-p(y, y)$

$p^{w}(x, y)=p(x, y)-\min \{p(x, x), p(y, y)\}$

olarak tanımlanan dönüşümler $X$ üzerinde birer metriktir (Matthew, 1994).

\subsection{Tanım}

$(X, p)$ kısmi metrik uzay ve $\left\{x_{n}\right\}, X$ de bir dizi olsun. (i) $\left\{x_{n}\right\}$ dizisinin bir $x \in X$ noktasina yakınsamas1 için gerek ve yeter şart $\lim _{n \rightarrow \infty} p\left(x, x_{n}\right)=p(x, x)$ olmasidir.

(ii) $\lim _{n, m \rightarrow \infty} p\left(x_{n}, x_{m}\right)$ limiti var ve sonlu ise $\left\{x_{n}\right\}$ dizisine Cauchy dizisi denir.

(iii) $X$ deki her $\left\{x_{n}\right\}$ Cauchy dizisi yakınsak ise yani $x \in X$ için $\lim _{n, m \rightarrow \infty} p\left(x_{n}, x_{m}\right)=p(x, x)$ oluyorsa $X$ tamdir denir.

(iv) Her $\varepsilon>0$ için en az bir $\delta>0$ var öyle ki $f\left(B\left(x_{0}, \delta\right)\right) \subset B\left(f\left(x_{0}, \varepsilon\right)\right)$ oluyorsa $f: X \rightarrow X$ dönüşümüne süreklidir denir (Matthew 1994).

\subsection{Lemma}

$(X, p)$ kısmi metrik uzay olsun.

(i) $(X, p)$ de bir $\left\{x_{n}\right\}$ dizisinin Cauchy dizisi olması için gerek yeter şart $\left(X, p^{S}\right)$ metrik uzayında Cauchy dizisi olmasidır.

(ii) $(X, p)$ kısmi metrik uzayının tam olması için gerek ve yeter koşul $\left(X, p^{S}\right)$ metrik uzayının tam olmasidir.

(iii) $\lim _{n \rightarrow \infty} p^{s}\left(x_{n}, x\right)=0 \Leftrightarrow p(x, x)=$ $\lim _{n \rightarrow \infty} p\left(x_{n}, x\right)=\lim _{n, m \rightarrow \infty} p\left(x_{m}, x_{n}\right) \operatorname{dir}$ (Matthew, 1994).

\subsection{Lemma}

$(X, p) \quad$ k1smi metrik uzay olsun. $\lim _{n \rightarrow \infty} p\left(x_{n}, x\right)=p(x, x)=0$ ise her $y \in X$ için, $\lim _{n \rightarrow \infty} p\left(x_{n}, x\right)=p(x, y)$ dir (Karapınar, 2011).

Diğer taraftan, (E.A)-özelliği, Aamri ve Moutawakil (2002) tarafindan metrik uzaylarda tanımlanmış ve Nazir ve Abbas (2014) tarafından kısmi metrik uzaylara aktarılmıştır. Bu çalışmada, integral tipli büzülme, kısmi metrik uzaylarda (E.A)-özelliğini sağlayan dönüşümler için tanımlanacaktır. (E-A)-özelliği sayesinde uzayın tamlığına ihtiyaç duyulmadan sabit noktanın varlığı ve tekliği ispatlanacaktır.

\subsection{Tanım}

$(X, p)$ k1smi metrik uzay ve $f, g: X \rightarrow X$ iki dönüşüm olsun.

(i) $X$ de bir $\left\{x_{n}\right\}$ dizisi için $\left\{f x_{n}\right\}$ ve $\left\{g x_{n}\right\}$ bir $t \in X \quad$ noktasina yakınsak iken 
$\lim _{n \rightarrow \infty} p\left(f g x_{n}, g f x_{n}\right)=p(t, t)$ ise $f$ ve $g$ dönüşümlerine bağdaşıktır denir.

(ii) $X$ de bir $\left\{x_{n}\right\}$ dizisi için $\left\{f x_{n}\right\}$ ve $\left\{g x_{n}\right\}$ bir $t \in X \quad$ noktasina yakınsak iken $\lim _{n \rightarrow \infty} p\left(f g x_{n}, g f x_{n}\right)$ limiti yok ise $f$ ve $g$ dönüşümlerine bağdaşık olmayan dönüşümlerdir denir (Nazir ve Abbas, 2014).

\subsection{Tanım}

$(X, p)$ kısmi metrik uzay ve $f, g: X \rightarrow X$ iki dönüşüm olsun. $X$ de bir $\left\{x_{n}\right\}$ dizisi için $\left\{f x_{n}\right\}$ ve $\left\{g x_{n}\right\}$ bir $t \in X$ noktasina yakınsak ise $f$ ve $g$ dönüşümleri (E.A)-özelliğini sağlar denir (Nazir ve Abbas 2014).

\subsection{Tanım}

$X \neq \varnothing \quad$ ve $f, g: X \rightarrow X$ iki dönüşüm olsun. Herhangi bir $x \in X$ için, $f x=g x$ ise $x$ noktasına ise $f$ ve $g$ dönüşümlerinin çakışık noktası denir. $f$ ve $g$ dönüşümleri çakışık noktada değişmeli ise $f$ ve $g$ zayıf bağdaşabilir dönüşümlerdir denir (Jungck, 1986).

\section{Sabit Nokta Teoremleri}

\subsection{Teorem}

$(X, p)$ kısmi metrik uzay ve $f, g, h, k: X \rightarrow X$ dört dönüşüm olmak üzere $f(X) \subseteq k(X)$ ve $g(X) \subseteq$ $h(X) \quad$ olsun. $\quad \omega: \mathbb{R}^{+} \rightarrow \mathbb{R}^{+} \quad$ Lebesque integrallenebilir, toplanabilir ve her $\eta>0$ için

$\int_{0}^{\eta} \omega(t) d t>0$ özelliğini sağlayan fonksiyon ve $\psi, \Phi$ uzaklığı değiştiren fonksiyonlar olmak üzere, her $x, y \in X$ için

$M(x, y)=$ $\max \left\{p(h x, k y), p(f x, h x),, p(g y, k y), \frac{p(h x, g y)+p(f x, k y,)}{2}\right\}$

iken

$$
\begin{aligned}
& \psi\left(\int_{0}^{p(f x, g y)} \omega(t) d t\right) \leq \psi\left(\int_{0}^{M(x, y)} \omega(t) d t\right)- \\
& \Phi\left(\int_{0}^{M(x, y)} \omega(t) d t\right)
\end{aligned}
$$

sağlansin.

(i) $f(X), g(X), k(X)$ veya $h(X)$ kümesi $X$ in kapalı bir altkümesi ve

(ii) $\{f, h\}$ veya $\{g, k\}$ çifti (E.A)-özelliğini sağlarsa

$\{f, h\}$ veya $\{g, k\}$ çakışık noktaya sahiptir. Ayrıca, $\{f, h\}$ veya $\{g, k\}$ zayıf bağdaşabilir ise $f, g, h$ ve $k$ dönüşümlerinin tek bir ortak sabit noktas1 vardir.

\section{Isspat}

I. Adım. $\{f, h\}$, (E.A)-özelliğini sağlasın. Bu durumda $X$ de bir $\left\{x_{n}\right\}$ dizisi ve $r \in X$ için $\lim _{n \rightarrow \infty} f x_{n}=\lim _{n \rightarrow \infty} h x_{n}=r$ dir. $\quad f(X) \subseteq$ $k(X)$ olduğundan, $f x_{n}=k y_{n}$ olacak şekilde $X$ de bir $\left\{y_{n}\right\}$ dizisi vardır. Böylece,

$\lim _{n \rightarrow \infty} f x_{n}=\lim _{n \rightarrow \infty} h x_{n}=\lim _{n \rightarrow \infty} k y_{n}=r$

yazılır. $\lim _{n \rightarrow \infty} g y_{n}=r$ olduğunu gösterelim. $\lim _{n \rightarrow \infty} g y_{n}=s$ olsun. (6) eşitsizliğinden,

$\psi\left(\int_{0}^{p\left(f x_{n}, g y_{n}\right)} \omega(t) d t\right) \leq \psi\left(\int_{0}^{M\left(x_{n}, y_{n}\right)} \omega(t) d t\right)-\Phi\left(\int_{0}^{M\left(x_{n}, y_{n}\right)} \omega(t) d t\right)$

olup burada

$M\left(x_{n}, y_{n}\right)=\max \left\{p\left(h x_{n}, k y_{n}\right), p\left(f x_{n}, h x_{n}\right), p\left(g y_{n}, k y_{n}\right), \frac{p\left(f x_{n}, k y_{n}\right), p\left(h x_{n}, g y_{n}\right)}{2}\right\}$

dir. (8) de $n \rightarrow \infty$ için limit alınırsa,

$\psi\left(\int_{0}^{p(r, s)} \omega(t) d t\right) \leq \psi\left(\int_{0}^{p(r, s)} \omega(t) d t\right)-\Phi\left(\int_{0}^{p(r, s)} \omega(t) d t\right)$

ve böylece $\Phi\left(\int_{0}^{p(r, s)} \omega(t) d t\right) \leq 0$ elde edilir.

$\Phi$ fonksiyonunun tanımından $\int_{0}^{p(r, s)} \varphi(t) d t=0$ ve $p(r, s)=0$ olup $s=r$ dir. Böylece, $\lim _{n \rightarrow \infty} g y_{n}=r$ dir.

$k(X)$ kümesi $X$ in kapalı alt kümesi olsun. Bu durumda, $k u=r$ olacak şekilde bir $u \in X$ vardır. (6) dan 
$\psi\left(\int_{0}^{p\left(f x_{n}, g u\right)} \omega(t) d t\right) \leq \psi\left(\int_{0}^{M\left(x_{n}, u\right)} \omega(t) d t\right)-\Phi\left(\int_{0}^{M\left(x_{n}, u\right)} \omega(t) d t\right)$

yazılır ve burada

$M\left(x_{n}, u\right)=\max \left\{p\left(h x_{n}, k u\right), p\left(f x_{n}, h x_{n}\right), p(g u, k u), \frac{p\left(f x_{n}, k u\right), p\left(h x_{n}, g u\right)}{2}\right\}$

dir. (11) de $n \rightarrow \infty$ için limit alınırsa,

$\psi\left(\int_{0}^{p(r, g u)} \omega(t) d t\right) \leq \psi\left(\int_{0}^{p(r, g u)} \omega(t) d t\right)-\Phi\left(\int_{0}^{p(r, g u)} \omega(t) d t\right)$

ve böylece $\Phi$ fonksiyonunun tanımından $\int_{0}^{p(r, g u)} \omega(t) d t=0$ ve $g u=r$ elde edilir. $u$ noktası $g$ ve $k$ dönüşümlerinin çakışık noktasıdır.

Şimdi, $g(X) \subseteq h(X)$ sağlandığından, $g u=h v=r$ olacak şekilde bir $v \in X$ vardır. $f v=r$ olduğunu gösterelim. (6) dan,

$\psi\left(\int_{0}^{p(f v, g u)} \omega(t) d t\right) \leq \psi\left(\int_{0}^{M(v, u)} \omega(t) d t\right)-\Phi\left(\int_{0}^{M(v, u)} \omega(t) d t\right)$

yazılır ve burada

$$
\begin{aligned}
M(v, u) & =\max \left\{p(h v, k u), p(f v, h v), p(g u, k u), \frac{p(f v, k u), p(h v, g u)}{2}\right\} \\
& =\max \left\{p(r, r), p(f v, r), p(r, r), \frac{p(f v, r)+p(r, r)}{2}\right\}=p(f v, r)
\end{aligned}
$$

olup, (14) den

$\psi\left(\int_{0}^{p(f v, r)} \omega(t) d t\right) \leq \psi\left(\int_{0}^{p(f v, r)} \omega(t) d t\right)-\Phi\left(\int_{0}^{p(f v, r)} \omega(t) d t\right)$

elde edilir. Sonuç olarak, $p(f v, r)=0$ olup $f v=r$ bulunur ve $k u=g u=h v=f v=r$ olur. $\{f, h\}$ ve $\{g, k\}$ zayıf bağdaşabilir ise $f r=h r$ ve $g r=k r$ 'dır.

II. Adım. $r$ noktasının $f, g, h$ ve $k$ dönüşümlerinin ortak sabit noktası olduğunu gösterelim. (6) dan

$$
\psi\left(\int_{0}^{p(f r, r)} \omega(t) d t\right)=\psi\left(\int_{0}^{p(f r, g u)} \omega(t) d t\right) \leq \psi\left(\int_{0}^{M(r, u)} \omega(t) d t\right)-\Phi\left(\int_{0}^{M(r, u)} \omega(t) d t\right)
$$

olup burada

$$
\begin{aligned}
M(r, u) & =\max \left\{p(h r, k u), p(f r, h r), p(g u, k u), \frac{p(f r, k u), p(h r, g u)}{2}\right\} \\
& =\max \left\{p(f r, r), p(f r, f r), p(r, r), \frac{p(f r, r), p(r, f r)}{2}\right\}=p(f r, r) .
\end{aligned}
$$

Böylece,

$\psi\left(\int_{0}^{p(f r, r)} \omega(t) d t\right) \leq \psi\left(\int_{0}^{p(f r, r)} \omega(t) d t\right)-\Phi\left(\int_{0}^{p(f r, r)} \omega(t) d t\right)$

ve $\Phi\left(\int_{0}^{p(f r, r)} \omega(t) d t\right) \leq 0$ elde edilir. Sonuç olarak, $f r=h r=r$ ve benzer olarak $g r=k r=r$ elde edilir. 
III. Adım. $f, g, h$ ve $k$ dönüşümlerinin ortak sabit noktasının tek olduğunu gösterelim. Aksini kabul ederek, $z$ noktası, $f, g, h$ ve $k$ dönüşümlerinin başka bir ortak sabit noktası olsun yani $f z=g z=k z=h z$ ve $z \neq r$ olsun. (6) dan

$\psi\left(\int_{0}^{p(r, z)} \omega(t) d t\right)=\psi\left(\int_{0}^{p(f r, g z)} \omega(t) d t\right) \leq \psi\left(\int_{0}^{M(r, z)} \omega(t) d t\right)-\Phi\left(\int_{0}^{M(r, z)} \omega(t) d t\right)$

ve

$$
\begin{aligned}
M(r, z) & =\max \left\{p(h r, k z), p(f r, h r), p(g z, k z), \frac{p(f r, k z), p(h r, g z)}{2}\right\} \\
& =\max \left\{p(r, z), p(r, r), p(z, z), \frac{p(r, z), p(r, z)}{2}\right\}=p(r, z) .
\end{aligned}
$$

Sonuç olarak,

$\psi\left(\int_{0}^{p(r, z)} \omega(t) d t\right) \leq \psi\left(\int_{0}^{p(r, z)} \omega(t) d t\right)-\Phi\left(\int_{0}^{p(r, z)} \omega(t) d t\right)$ ve $\Phi\left(\int_{0}^{p(r, z)} \omega(t) d t\right) \leq 0$

elde edilir. Böylece, $p(r, z)=0$ ve dolayısıyla $r=z^{\prime}$ dir.

\subsection{Sonuç}

$(X, p)$ kısmi metrik uzay ve $f, h, g, k: X \rightarrow X$ dört dönüşüm olmak üzere $f(X) \subseteq k(X)$ ve $g(X) \subseteq h(X)$ olsun. $\omega: \mathbb{R}^{+} \rightarrow \mathbb{R}^{+}$Lebesque integrallenebilir, toplanabilir ve her $\eta>0$ için

$\int_{0}^{\eta} \omega(t) d t>0$

koşulunu sağlayan fonksiyon ve $\psi, \Phi$ uzaklığı değiştiren fonksiyonlar olmak üzere, her $x, y \in X$ için

$$
\begin{aligned}
& M(x, y)=\max \left\{p(h x, k y), p(f x, h x,), p(g y, k y), \frac{p(h x, g y)+p(f x, k y)}{2}\right\} \text { iken }, \\
& \int_{0}^{p(f x, g y)} \omega(t) d t \leq \int_{0}^{M(x, y)} \omega(t) d t-\Phi\left(\int_{0}^{M(x, y)} \omega(t) d t\right)
\end{aligned}
$$

sağlansin.

(i) $f(X), h(X), g(X)$ veya $k(X)$ kümesi $X$ in kapalı bir altkümesi ve

(ii) $\{f, h\}$ veya $\{g, k\}$ çifti (E.A)-özelliğini sağlarsa

$\{f, h\}$ veya $\{g, k\}$ çakışık noktaya sahiptir. Ayrıca, $\{f, h\}$ veya $\{g, k\}$ zayıf bağdaşabilir ise $f, h, g$ ve $k$ dönüşümlerinin tek bir ortak sabit noktası vardır.

\subsection{Sonuç}

$(X, p)$ kısmi metrik uzay ve $f, k: X \rightarrow X$ iki dönüşüm olmak üzere $f(X) \subseteq k(X)$ olsun.. $\omega: \mathbb{R}^{+} \rightarrow \mathbb{R}^{+}$ Lebesque integrallenebilir, toplanabilir ve her $\eta>0$ için

$\int_{0}^{\eta} \omega(t) d t>0$

koşulunu sağlayan fonksiyon ve $\psi, \Phi$ uzaklığı değiştiren fonksiyonlar olmak üzere, her $x, y \in X$ için

$M(x, y)=\max \left\{p(k x, k y), p(f x, k x),, p(f y, k y), \frac{p(k x, f y)+p(f x, k y)}{2}\right\}$ iken

$\psi\left(\int_{0}^{p(f x, f y)} \omega(t) d t\right) \leq \psi\left(\int_{0}^{M(x, y)} \omega(t) d t\right)-\Phi\left(\int_{0}^{M(x, y)} \omega(t) d t\right)$

sağlansın. 
(i) $f(X)$ veya $k(X)$ kümesi $X$ in kapalı bir altkümesi ve

(ii) $\{f, k\}$ çifti (E.A)-özelliğini sağlarsa

$\{f, k\}$ çakışık noktaya sahiptir. Ayrıca, $\{f, k\}$ zayıf bağdaşabilir ise $f$ ve $k$ dönüşümlerinin tek bir ortak sabit noktası vardır.

\section{4. Örnek}

$X=\{0,1,2,3, \ldots\}$ olmak üzere, $p: X \times X \rightarrow\left[0, \infty\left[, \quad p(x, y)=\left\{\begin{array}{c}2, \\ \max \{x, y\},\end{array} \begin{array}{c}(x, y)=(3,0) \\ \text { diğer }\end{array}\right.\right.\right.$ kuralıyla verilsin. Ayrıca, $\psi, \Phi:\left[0, \infty\left[\rightarrow\left[0, \infty\left[\right.\right.\right.\right.$ ve $\omega: \mathbb{R}^{+} \rightarrow \mathbb{R}^{+}$fonksiyonları $\psi(x)=4 x \quad \Phi(x)=\sqrt{x}$ ve $\omega(x)=$ $2 x$ olarak tanımlansin. $f, h, g, k: X \rightarrow X$

$f(x)=\left\{\begin{array}{cc}0, & x=0 \\ 2 x+1 & x \neq 0\end{array}, \quad h(x)=\left\{\begin{array}{cc}0, & x=0 \\ 3 x & x \neq 0\end{array}, \quad g(x)=0, \quad k(x)=x\right.\right.$ olarak tanımlanırsa

i) $f(X) \subseteq k(X)$ ve $g(X) \subseteq h(X)$ dir.

ii) $g(X)$ kapalıdır.

iii) $\{f, h\}$ dönüşümleri (E.A)-özelliğini sağlar ve bağdaşık değildir: $x_{n}=1$ dizisi için $f x_{n} \rightarrow 3$ ve $h x_{n} \rightarrow 3$ olup 2.9. Tanımdan dolayı $\{f, h\}$ dönüşümleri (E.A)-özelliğini sağlar.

iv) (6) şartını göstermek için:

$x=0$ ve her $y \in X$ için (6) sağlanır.

$x=1$ ve her $y \in X$ için,

$$
\begin{array}{r}
\psi\left(\int_{0}^{p(f x, g y)} \omega(t) d t\right)=\psi\left(\int_{0}^{p(3,0)} 2 t d t\right)=4.2^{2} \leq 4.3^{2}-3 \\
=\psi\left(\int_{0}^{p(f x, h x)} 2 t d t\right)-\Phi\left(\int_{0}^{p(f x, h x)} 2 t d t\right)
\end{array}
$$

olup (6) şartı sağlanır.

$x>1$ ve her $y \in X$ için,

$$
\begin{aligned}
& \left(\psi \int_{0}^{p(f x, g y)} \omega(t) d t\right)=\psi\left(\int_{0}^{2 x+1} 2 t d t\right)=4(2 x+1)^{2} \leq 4 .(3 x)^{2}-3 x \\
& =\psi\left(\int_{0}^{p(f x, h x)} 2 t d t\right)-\Phi\left(\int_{0}^{p(f x, h x)} 2 t d t\right) \leq \psi\left(\int_{0}^{M(x, y)} \omega(t) d t\right)-\Phi\left(\int_{0}^{M(x, y)} \omega(t) d t\right)
\end{aligned}
$$

elde edilir ve (6) şartı sağlanır. Böylece 3.1 Teoreminin bütün koşulları sağlanır. Ayrıca, $f(0)=g(0)=$ $h(0)=k(0)=0$ olup $x=0$ noktas $1 f, g, h, k$ dönüşümlerinin tek ortak sabit noktasıdır.

\section{Sonuç}

Matematikte pek çok problemin çözümünde kimi zaman tam sonucu bulmak mümkün değildir. $\mathrm{Bu}$ bakımdan varlık ve teklik problemlerinin çözümünde Banach büzülme prensibi önemli bir rol oynar. Banach büzülme prensibinde uzayın tam olması ve dönüşümün sürekli olması sabit noktanın varlığını göstermek açısından önemlidir.
Gerek uzay gerekse dönüşümün özellikleri değiştirilerek Banach büzülme prensibinin literatürde pek çok genelleştirmesi verilmiştir. Bu çalışmada, metrik uzaylardan daha genel olan kısmi metrik uzaylar üzerinde (E.A)-özelliğini sağlayan dört dönüşüm için integral tip büzülme tanımlanmıştır. (E.A)-özelliği sayesinde uzayın tam olması gerekmeksizin dönüşümlerin ortak sabit noktasının varlığı gösterilmiştir. 


\section{Kaynaklar}

Aamri, M. ve El-Moutawakil, D., 2002. Some new common fixed point theorems under strict contractive conditions. Journal of Mathematical Analysis And Applications, 270, 181-188.

Abdeljawad, T., 2011. Fixed points for generalized weakly contractive mappings in partial metric spaces. Mathematical and Computer Modelling, 54, 2923-2927.

Altun, I., Sola, F. ve Simsek, H., 2010. Generalized contractions on partial metric spaces. Topology And Its Applications, 157, 2778-2785.

Babu, G.V.R., Lalitha, B. ve Sandhya, M.L., 2007. Common fixed point theorems involving two generalized altering distance functions in four variables. Prooceding Of The Jangjeon Mathematical Society, 10 (1), 83-93.

Banach, S., 1922. Sur les oprations dans les ensembles abstraits et leur applications aux equations integrales. Fundemental Mathematics, 3, 133-181.

Branciari, A., 2002. A fixed point theorem for mappings satisfying a general contractive condition of integral type. International Journal of Mathematics and Mathematical Sciences, 29 (9), 531-536.

Dutta, P.N. ve Binayak, S.C., 2008. A generalisations of contraction principle in metric spaces, Fixed Point Theory and Appl., 2008, Article ID 406368.

Jungck, G., 1986. Compatible mappings and common fixed points. Int. J. Math. Sci., 9, 771-779.
Kadelburg, Z., Nashine, H.K. ve Radenovic, S. 2013. Fixed point results under various contractive conditions in partial metric spaces. Revista de la Real Academia de Ciencias Exactas, Fisicas y Naturales. Serie A. Matematicas, 107 (2), 241-256.

Karapinar, E., 2011. Generalizations of Caristi Kirk's theorem on partial metric spaces, Fixed Point Theory and Applications, 2011, Article ID 4.

Matthews, S.G., 1992. Partial Metric Topology, Research Report 212, Department of Computer Science University Of Warwick.

Matthews, S.G., 1994. Partial Metric topology in Proceedings of the 8th Summer Conference at Queens College,S. Andima, Ed., 728 of Papers on General Topology and Applications, Annals of the New York Academy of Sciences 183-197.

Naidu, S.V.R., 2003. Some fixed point theorems in metric spaces by altering distances. Czechoslovak Mathematical Journal, 53 (1), 205-212.

Nazir, T. ve Abbas, M., 2014. Common fixed points of two pairs of mappings satisfying (E.A)-property in partial metric spaces. J. Inequal. Appl. 2014, Article ID 23.

Rhoades, B.E., 2001. Some theorems on weakly contractive maps. Nonlinear Analysis: Theory, Methods and Applications, 47 (4), 2683-2693.

Turkoglu, D. ve Ozturk, V., 2012. Common fixed point results for four mappings on partial metric spaces. Abstract And Applied Analysis, Article ID 190862.

Valero, O., 2005. On Banach fixed point theorems for partial metric spaces. Applied General Topology, 6 (2), 229-240. 